\title{
Semantic Ontology-Based Strategy for Image Retrieval in Conceptual Modelling
}

\author{
Simon McGinnes \\ School of Computer Science and Statistics, Trinity College Dublin, Ireland \\ Simon.McGinnes@tcd.ie
}

\begin{abstract}
A strategy is described which integrates the use of keyword matching, thesaurus and a simple ontology to retrieve images. The strategy was developed to support a conceptual modelling technique that depicts concepts using images, in which searching must integrate tightly into the modelling process without interrupting the train of thought. Suggested by cognitive mechanisms, the strategy varies semantic constraints over time to tune results. It exploits commonalities between modelling and searching tasks to minimise cognitive task-switching penalties and to maintain momentum in modelling sessions. Aside from its use in modelling, the strategy has potential applications in semantic search and information retrieval more broadly, where identification of underlying concepts may help to increase the relevance of search results.
\end{abstract}

Keywords: Semantic search; semantic retrieval; image search; conceptual modelling; ontologies; database design.

\section{Introduction}

Conceptual modelling (CM) is an expert task, and conceptual models can be hard to understand. One way of making models more understandable is to use images (Fig. 1.); this practice facilitates recognition, reducing the need for slow and cognitively-intensive interpretation and searching. Well-chosen symbols convey meaning effortlessly, by triggering mental associations. Most CM techniques use "box-andline" notations. UML, for example, depicts classes as rectangles, regardless of what they represent. But this makes it harder to differentiate between items, so a valuable channel for conveying meaning is lost. The BCM technique [1] instead depicts each entity type with its own image, which the modeller may choose using a suitable search facility. In this context, image search must be fast and require little interaction, to maintain a free flow of ideas; task-switching incurs a heavy cognitive penalty. Relevant results should be returned earliest and distractions should be avoided. Every image found for a given entity type should correspond to its related mental concept.

Image search techniques that rely on text matching alone can be overly sensitive to choice of search terms, synonyms, homonyms and so on. The lack of semantic context creates a gap between the coding of images and the searcher's intentions. Various ways of adding semantics to image search have been tested. For example, one technique places images in $n$-dimensional semantic space and maps them onto specified context planes [2]; as in many techniques, the images must be tagged in advance, 
which can be onerous. Machine interpretation of images would facilitate rapid tagging but remains insufficiently reliable, in the general case. Certain semantics can be inferred, however. Some techniques use hyperlinks as a proxy for semantic associations; others tag images according to similarity to exemplar images, or try to infer the searcher's domain of reference [3-4].

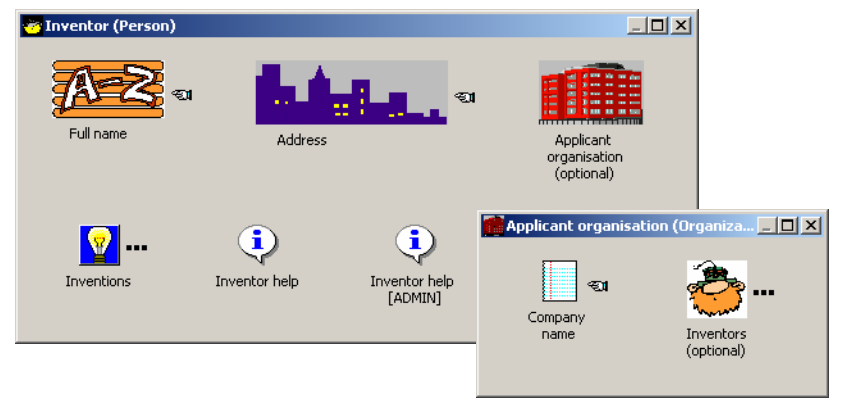

Fig. 1. A BCM model fragment. Each image symbolises an entity type (mental concept); the presence of an image in a window represents a relationship between entity types

\section{Search Strategy}

Our search strategy is loosely inspired by aspects of cognition. We apply a simple single-level ontology of archetypal categories, reflecting innate concepts such as people, places and activities, and pitched at an "everyday" level to minimise cognitive effort [5-6]. Associative recall is emulated with keyword matching and memory with a bank of images, each linked to an archetypal category and search terms. Lateral thinking, where one idea leads to another, is invaluable in CM; we emulate it using a thesaurus containing linked terms. We use segregated processing and competition; different search steps are handled by separate threads and the outputs are combined.

The search function is invoked when a user adds a new entity type to a model. In BCM, each entity type is named and corresponds to a particular domain concept. It is also associated with an archetypal category from the list people, places, documents, organisations, activities, physical objects, conceptual objects, systems and categories. Although it is very simple, when used in CM this single-level ontology has been found to reduce modellers' skill requirements and improve model quality [1]. The search function is therefore supplied with two values: (a) an initial search term, which is the name of an entity type, and (b) the associated archetypal category. The aim is to use search term and category to find one or more relevant images.

The process is progressive (Table 1). In steps 1 and 2, exact or near-exact matches are sought for the search term and category. For common or very general concepts, such as customer, this will typically retrieve a variety of images (Fig. 2). However, for unusual concepts (e.g. inventor) exact matches are less likely. The search proceeds in steps 3 and 4 to find images that match the search term but not the category. The purpose is to find images that do not represent the concept itself, but may nonetheless symbolise it. In Fig. 1., applicant organisation is depicted as a building; obviously, an organisation is not a building, but the building can represent an organisation because 
the ideas are closely linked. For the same reason the abstract concept bank account could be depicted with the image of an ATM card. The term "analogy" is used in Table 1 as shorthand for this type of relationship between image and concept.

Table 1. Example of search algorithm for entity type customer rep (category person)

\begin{tabular}{|c|c|c|c|}
\hline Step & Category & Terms sought & Images returned \\
\hline 1. & \multirow{2}{*}{ people } & customer rep & Exact matches only \\
\hline 2. & & customer or rep & Near-exact matches \\
\hline 3. & \multirow{2}{*}{ not people } & customer rep & Close analogies/related ideas \\
\hline 4. & & customer or rep & Near analogies/related ideas \\
\hline 5. & people & \multirow{2}{*}{$\begin{array}{l}\text { Thesaurus terms matching customer } \\
\text { rep, customer or rep }\end{array}$} & \multirow{2}{*}{$\begin{array}{l}\text { Synonyms, closely-related } \\
\text { concepts and their analogies }\end{array}$} \\
\hline 6. & not people & & \\
\hline 7. & people & \multirow{2}{*}{$\begin{array}{l}\text { Repeated recursively using the terms } \\
\text { from thesaurus matching those from } \\
5 .\end{array}$} & \multirow{2}{*}{$\begin{array}{l}\text { Successively less-closely- } \\
\text { related concepts \& analogies }\end{array}$} \\
\hline 8. & not people & & \\
\hline 9. & people & Any & Loose matches (any people) \\
\hline
\end{tabular}

In Steps 5 and 6, images are sought for thesaurus terms linked to the search term; each linked term may represent a synonym or a related idea. The process continues asynchronously in steps 7 and 8, traversing links recursively. The result is akin to spreading activation and produces a progressively widening set of results. Relevance gradually decreases as the semantic net widens. Competition is achieved by giving preference to images retrieved most often and via the shortest semantic paths.

Concurrently (step 9), the search displays any other images matching the category. Even if no suitable images were previously found, some broadly-relevant images will

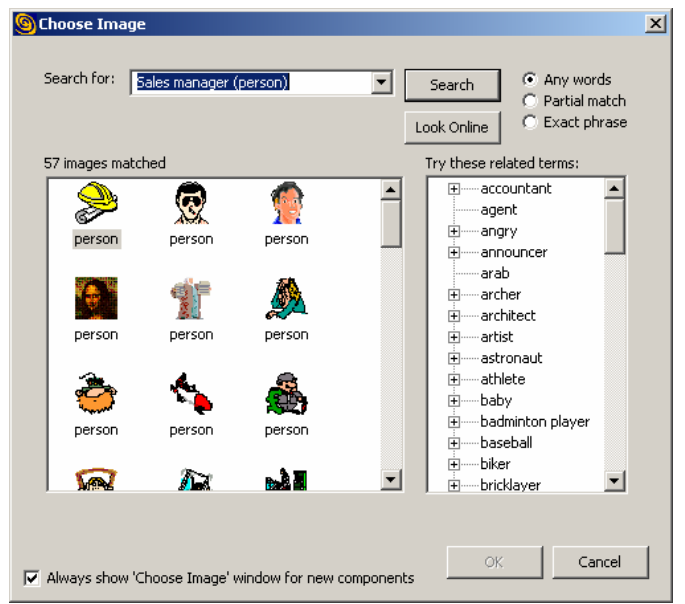

Fig. 2. Prototype image search facility 
now be displayed; this is particularly helpful for unusual concepts. The algorithm continues until termination criteria are met or the user interrupts the search. The strategy is not designed to find optimal results; instead, potential solutions are offered and the user exercises subjective judgment, as in satisficing. The modeller benefits since each image is related to the original concept, if only tenuously, and the mind is adept at identifying links. For example, in Fig. 1. inventions is depicted as a light bulb (which was found because it is linked with idea, which in turn is linked to invention).

The search strategy was implemented in a tool supporting BCM and tested in group sessions [1]. Designed to exploit synergies between modelling and searching, the tool performed well enough to integrate seamlessly into the modelling process. As anticipated, the most relevant images were displayed earliest and (crucially) looser matches were provided as well. This allowed the modeller to proceed even if he/she intended to search later for a better image. As hoped, synonyms were found automatically and homonyms were less of a problem than with pure keyword search, because the archetypal category automatically disambiguated ambiguous terms.

\section{Conclusion and Further Work}

This research suggests that benefits might be obtained in ontology-based search by giving preference to certain basic-level categories. It also demonstrates the effects of relaxing semantic constraints during search. The ontology was a simple one, but any more complex ontology could be used, and experimentation with a more complex ontology might permit more sophisticated tuning. In the context of CM, the strategy could go further. For example, the content of the conceptual model might beneficially be used in a similar way to the thesaurus. For the strategy to be useful in more general semantic search, large-scale tagging of content would be required; while automated tagging remains a challenge, one possible solution might be to crowdsource the task.

\section{References}

1. McGinnes, S., Amos, J.: Accelerated Business Concept Modeling: Combining User Interface Design with Object Modeling. In: Harmelen, M.V., Wilson, S. (eds.) Object Modeling and User Interface Design: Designing Interactive Systems, pp. 3-36. Addison Wesley, London (2001)

2. Kawamoto, M., Kiyoki, Y., Yoshida, N., Fujishima, S., Aiso, S.: An Implementation of a Semantic Associative Search Space for Medical Document Databases. In: SAINT 2004 Workshops, International Symposium on Applications and the Internet, pp. 488-493. IEEE Computer Society, Tokyo (January 26-30, 2004)

3. Xu, X., Lee, D.J., Antani, S., Long, L.R.: A Hybrid Approach for Online Spine X-ray Image Retrieval Based on CBIR and Relevance Feedback. IEEE Transactions on Information Technology in Biomedicine, 1-10 (2005)

4. Zhuge, H.: Retrieve images by understanding semantic links and clustering image fragments. The Journal of Systems and Software 73, 455-466 (2004)

5. Moore, C.J., Price, C.J.: A Functional Neuroimaging Study of the Variables that Generate Category-Specific Object Processing Differences. Brain 122, 943-962 (1999)

6. Pansky, A., Koriat, A.: The Basic-Level Convergence Effect in Memory Distortions. Psychological Science 15, 52-59 (2004) 